\title{
"Grow old along with me! The best is yet to be"
}

\author{
Contesting Aging \& Loss \\ Janice E. Graham and \\ Peter H. Stephenson, editors \\ University of Toronto Press; 2010.
}

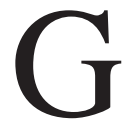
erontologists seem to divide themselves into two warring categories: poets versus pathologists. The former consider the phenomenon of aging as a part of life, a normal stage in human development, and often portray it as a positive process . For example, those who hold such views will characteristically quote the English poet Robert Browning: "Grow old along with me! The best is yet to be, the last of life, for which the first was made."

If the poets tend to be purveyors of the social sciences, for their part the pathologists are usually doctors or biologists. As far as they can see, aging is a negative process resulting in an increase in biological entropy, decreased reserve, a higher chance of illness and ultimately death. That being said, their laureate is of no less distinguished lineage, with Shakespeare outlining "Last scene of all, That ends this strange eventful history. ... Sans teeth, sans eyes, sans taste, sans everything."

Clearly, this book is a production of the poetic school and as the title suggests, contesting " ... the idea that much of what we experience as we age is simply inevitable and ... is meant to be a corrective based on ethnographic evidence."

The authors, mainly Canadians with a smattering of others, are all social scientists. They offer that we (mainly but not exclusively medical/health practitioners) for various social, psychological and economic reasons tend to pigeonhole the elderly as those who are on an inevitable downward spiral. Furthermore, we tend to act as if they inevitably suffer from ongoing and accumulating losses and have no or very poor coping mechanisms. The

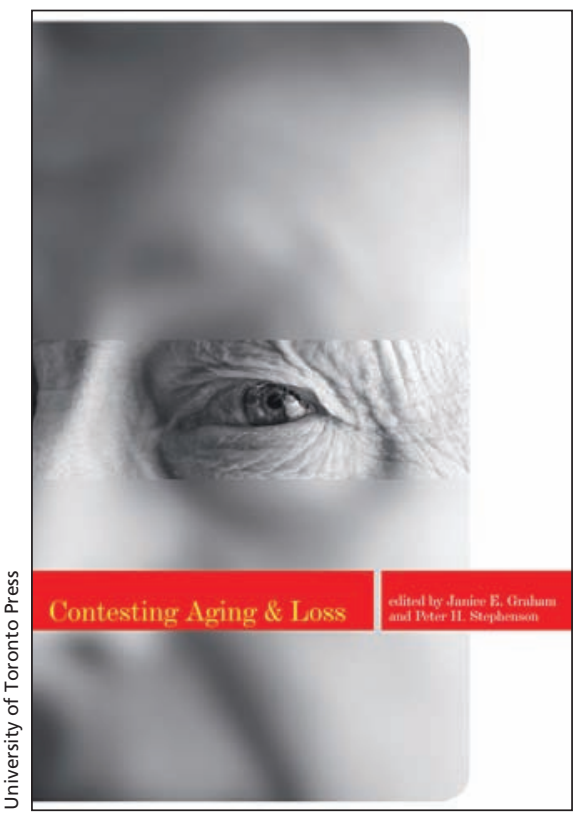

authors use, as mentioned above, an "ethnographic" technique, mostly via qualitative research and interviews to get to the bottom of elders' concerns and above all to demonstrate how so many of these folks actually cope reasonably well - at least as far as they themselves are concerned. The authors enjoin us to listen to the elders while at the same time parking our preconceptions and prejudices at the door.

To make their point, the researchers examine various populations such as elderly Italian Canadians, Dutch seniors and Australian lower income men living in inner city Sydney. The nine-page introduction by the two editors actually does an excellent job of summarizing the book's findings and of succinctly offering its conclusions. The busy clinician can just read this section and the text's main argument will become clear.

For all of its value, is this a book for doctors? Tough question. On one hand, we do need to hear the points of view alluded to above which might help us to counter our natural tendency to ageism. On the other, many clinicians will have some difficulty with this book. Much of the material is written in a dense and difficult social sciences style. For example, “... shows us that by privileging the notions of what counts as social capital (novelty, active responsiveness, outgoing behaviour, executive functions), we have built clinical markers that recognize and value self-aggrandizement (selling oneself) from the cradle to the grave." Or when culture is defined, we are told it “... involves processes and practices constantly occurring within power laden social contexts and locations to create fluid, contested, negotiable, ambiguous meanings ..."

Hmmmm.

From the purely scientific point of view, many of us might raise an eyebrow at some of the methodologies used. For example (and not to put down qualitative research per se), the chapter on attitudes among Italian Canadians, while actually in many ways quite interesting, is based on interviews of a total of 18 individuals in two provinces. It is difficult to justify generalization from this small, nonrandomized sample. As well, from time to time, the reader gets the feeling that the authors don't always think too much of the medical profession. For example, we are taken to task for testing certain neuropsychological functions as part of a cognitive assessment: "Personhood is not defined by being able to perform nonsensical cognitive tasks such as repeating once phone number backwards (which is really used as a measure in some tests)" (emphasis mine).

That being said, I thoroughly enjoyed the chapter entitled "The Science, Politics, and Everyday Life of Recognising Effective Treatments for Dementia" by one of the editors (JEG). As summarized in the introduction, the author " ... grapples with the corporate manipulations that attend Alzheimer's disease, demonstrating (ironically) how "soft" social science along with 
clinical research have actually been appropriated by the pharmaceutical industry to promote certain drugs that may or may not even actually help people to resist the disease in its early stages." Here here!

Despite the sections of the book which are not antidoctor, the kind of arch attitude ("really used") alluded to above will hardly encourage us to bridge the gap between C.P. Snow's Two Cultures. Science (in this case medical care of the elderly) and the humanities do need to speak to each other. This book, by eminent scholars in the latter discipline makes an important point. But the truth is that as a result of the turgid academic style (and I admit we physicians can be just as bad in our own scribblings), it will not be easy for doctors to take in and digest the valuable material on offer even if the intrepid reader succeeds in ploughing on to the end.
And more's the pity as the message is an important one to all of us who care about and for elderly persons and who will, we all hope, eventually reach old age ourselves.

\author{
A. Mark Clarfield MD \\ Soroka Hospital and Faculty of Health \\ Sciences \\ Ben-Gurion University \\ Negev, Israel
}

CMAJ 2011. DOI:10.1503/cmaj.101431 